\title{
Sudden DEATH AND EPILEPSY
}

\author{
Case-control Study of SUDEP \\ Langan Y, Nashef L, Sander JW \\ Neurology 2005;64:1131-1133
}

PURPOSE: To examine the influence of various factors on the risk of sudden unexpected death in epilepsy (SUDEP). METHODS: The authors investigated 154 cases in which a postmortem examination was performed. Each case had four controls with epilepsy from the community, matched for age and geographic location. Backward stepwise conditional logistic regression analysis was performed, and odds ratios for risk and protection were determined.

RESULTS: The risk of SUDEP was increased with a history of generalized tonic-clonic seizures in the previous 3 months (odds ratio [OR]: $13.8 ; 95 \% \mathrm{Cl}: 6.6$ to 29.1). The presence of supervision at night was found to be protective (OR: $0.4 ; 95 \% \mathrm{Cl}: 0.2$ to 0.8 ) when a supervising individual shared the same bedroom or when special precautions such as a listening device were used (OR: 0.1; 95\% CI: 0.0 to 0.3$)$.

CONCLUSIONS: This work lends support to the view that SUDEP is a seizure-related phenomenon and that control of tonic-clonic seizures is important in its prevention. Nocturnal supervision seems to protect against SUDEP.

\section{COMMENTARY}

$\mathrm{N}$ ow another article (published by Langan and colleagues) has appeared about SUDEP (sudden unexpected death in epilepsy). This topic is of great concern for neurologists treating patients who may die suddenly, typically during sleep. Whether to tell patients about the risk of sleep-associated death during a seizure is a real dilemma. Clinicians frequently try to reassure patients and relatives that seizures usually are not dangerous. For patients having many seizures, the thought of dying during a seizure could be a point of great anxiety and ruin the little quality of life that they enjoy. In contrast, if clinicians do not warn patients of the risk of SUDEP, they could learn about it, nevertheless, by searching the Internet. How can clinicians be forthcoming without evoking anxiety?

Langan and colleagues make a valuable contribution to resolving this problem. The investigators performed a community-based, case-control analysis of SUDEP to elucidate and narrow the risk factors. During a 9-year period, epilepsy patients (age range, 16 to 50 years) who died suddenly were identified throughout England and Wales. Contacting neurologists throughout the country and through patient organizations identified additional SUDEP cases. These cases were then matched with four control cases of living patients with epilepsy of the same age and geographic location. The authors then re-

Epilepsy Currents, Vol. 5, No. 6 (November/December) 2005 pp. 223-224 Blackwell Publishing, Inc.

( ) American Epilepsy Society viewed the medical records of both the control and SUDEP patients to extract data relevant to the study, including duration of epilepsy, seizure type and severity, control of seizures, antiepileptic (AED) drug history, family history of sudden death, learning disabilities, alcohol consumption, EEG changes, and supervision at night. The variables found to be most predictive of SUDEP were a history and frequent occurrence of generalized seizures as well as the use of several specific drugs, especially carbamazepine. Not surprisingly, supervision at night was protective. Very surprising, however, was the finding that having asthma seemed to be protective, which may be because of a protective effect of asthma medication, such as $\beta$-stimulating agents or cortisone. Before publication of this article, male gender was thought to be an important factor in the risk of SUDEP, but the finding was not confirmed by this study, in spite of there being an excess of male subjects.

Current polytherapy was not found to be an independent risk factor, as would be expected. In contrast, patients who had used a large number of AEDs, probably reflecting the patient's refractory state and seizure severity, were at greater risk of SUDEP. An increased risk also was found for patients who had never been administered an AED or who had undefined seizures, suggesting that patients should be encouraged to take AEDs for their seizures. Carbamazepine has been associated with many cases of SUDEP and can cause arrhythmia, as can phenytoin. Carbamazepine is the gold standard of treatment for partial seizures. Many epilepsy guidelines encourage, if not dictate, its use as a first-line choice, which means that carbamazepine is used more frequently than any other AED. Thus, it would follow that more patients with SUDEP would 
be taking carbamazepine than other AEDS. The role of carbamazepine in SUDEP, therefore, must be clarified further. Other factors that were believed to be involved in contributing to the risk of SUDEP but were not confirmed in this article include youth, remote symptomatic epilepsy, structural pathology, severe epilepsy, alcohol abuse, abnormal EEGs, mental handicap, use of psychopharmacologic agents, noncompliance with AED regimen, very fast alterations in AED therapy, low AED blood concentrations, and nocturnal seizures.

SUDEP usually occurs during the night, with no witness. Although the cause is still unclear, the main hypothesis is that the deaths occur during a seizure or postictally because of centrally induced respiratory insufficiency. Occasionally, the cause is a cardiac arrhythmia induced by a seizure (1).

So what can be done to alleviate the risk of SUDEP? An alarm would alert a caretaker as well as possibly serve to stimulate the patient and, perhaps, prohibit a pending respiratory depression or arrhythmia. Physicians encourage independent living; however, people who live alone and have frequent seizures might reconsider other arrangements.

More epidemiologic studies of SUDEP are needed to resolve the issues of risk and SUDEP, as the Langan et al. article is not definitive. Patients at risk of SUDEP should be evaluated by a cardiologist to detect a predisposition for arrhythmias; providing these patients with a simple pacemaker or defibrillator may be protective. Implanted devices allow Holter monitoring of the ECG for months at a time, which permits the opportunity to monitor a seizure in a natural setting. As a preventive treatment, for selected high-risk patients, the implantable cardioverter/defibrillator is very effective in terminating lifethreatening ventricular tachyarrhythmias and is known to be a cost-effective method of reducing mortality (2). The device would probably work as well for patients at risk for SUDEP. Taking the problem of SUDEP seriously and discussing it with patients that are at risk is the first step in its prevention.

by Elinor Ben-Menachem, $M D, P h D$

\section{References}

1. Camfield P, Camfield C. Sudden unexpected death in people with epilepsy: a pediatric perspective. Semin Pediatr Neurol 2005;12:1014

2. Lane RE, Cowie MR, Chow AWC. Prediction and prevention of sudden cardiac death in heart failure. Heart 2005;91:674-680. 